Brown, A. M. (1959). J. gen. Microbiol. 20, 532-539

\title{
Growth Idiosyncrasies of Some Bordetella pertussis Strains, and the Intracerebral Virulence of these Strains in Mice
}

\author{
By ANNIE M. BROWN \\ Serum Laboratory, Medical Research Council Laboratories, \\ Carshalton, Surrey
}

SUMMARY: The growth characteristics of eight strains of Bordetella pertussis were studied in defined liquid media. Two of these strains (1017, 1018), which are both very virulent on intracerebral injection into mice, were found to have growth idiosyncrasies. Both these strains differed from typical virulent pertussis strains and from a degraded strain in that they needed more magnesium and a different gas phase (carbon dioxide and oxygen) for optimal growth in defined liquid medium. Both strains were inhibited by crystalline lysozyme $(100 \mathrm{mg} . / \mathrm{ml}$.) in defined medium, whereas typical virulent strains and a degraded strain were insensitive to it.

The growth of Bordetella pertussis was reviewed by Rowatt (1957) who concluded that 'knowledge of the metabolism of these organisms is slight'. Conditions for their growth on solid media and in defined liquid media were discussed, but no reference was made to growth idiosyncrasies of strains which were highly virulent when injected intracerebrally into mice. The study of the intracerebral invasion in mice of two strains of B. pertussis (Brown, 1958) led to the conclusion that the shape of the growth curves obtained was determined by the balance between the growth in, and removal of the organisms from, the brain, and was characteristic for each strain. The following work is a study of the growth of some $B$. pertussis strains of determined intracerebral virulence in defined liquid media.

\section{METHODS}

Organisms. Eight strains of Bordetella pertussis were used: five smooth, virulent, freshly isolated strains (1011 (NCTC 7234), 1012 (NCTC 20948), 1013 (NCTC 21194) and 1014, 1015 from Dr B. W. Lacey), two smooth virulent strains (1017 described by Kendrick, Eldering, Dixon \& Misner, 1947, 1018 described by Andersen 1952; both were highly virulent when injected by the intracerebral route). One degraded strain $1011 \mathrm{HR}$ derived from strain 1011 by continuous subculture on Bordet-Gengou medium made with $50 \%(\mathrm{v} / \mathrm{v})$ horse blood.

Measurement of virulence of strains. The LD 50 dose for each strain determined in albino male Swiss bred mice weighing 17-20 g. in the manner described previously (Brown, 1958) was considered the measure of its virulence. Values obtained both by intracerebral injection and by intranasal instillation were the average of at least two results.

Plate counts of pertussis organisms. These were made by the method of 
Miles \& Misra (1938). No penicillin was added to the medium as knowledge of the presence of contaminants was essential.

Preparation of suspensions of organisms for the inoculation of growth experiments. The storage of organisms, and their recovery from the dried state for the preparation of suspensions, was as previously described (Brown, 1958). In this work all organisms were washed once with saline and resuspended in the medium described by Jebb \& Tomlinson (1955). The estimation of total numbers in each suspension was made by opacity measurements as before. Dilutions of suspensions which contained approximately $4 \times 10^{9}$ viable organisms $/ \mathrm{ml}$. were prepared, and unless otherwise stated the inoculation of cultures in growth experiments was approximately $4 \times 10^{3}$ organisms $/ \mathrm{ml}$. medium.

\section{Defined liquid medium and growth experiments}

Basal medium. The medium described by Jebb \& Tomlinson (1955) was found satisfactory for this work. It was essential to boil the medium during charcoal absorption or to stir efficiently when the process was done in the steamer. Uneven distribution of the charcoal during absorption produced a medium which did not appear to have a uniform composition. Nicotinamide (to $4 \times 10^{-7} \mathrm{M}$ ) and haemin ( $1 \mathrm{mg} . / \mathrm{ml}$; added as haematin) were the growth factors added. Magnesium sulphate was substituted for magnesium chloride in the medium.

Design of growth experiments. Volumes $(20 \mathrm{ml}$.) of basal medium were dispensed in $100 \mathrm{ml}$. hard glass square bottles which were closed with loose aluminium caps, and autoclaved for $10 \mathrm{~min}$. at $15 \mathrm{lb} . / \mathrm{sq}$.in. Additions of nicotinamide, haematin and any other substances were made under aseptic conditions immediately before inoculation. Two bottles of each variant of the medium were inoculated with every test strain of organism. The cultures were incubated on racks inclined at an angle of $45^{\circ}$ in a hot room at $36^{\circ}$. Illumination from behind with a strip light enabled the course of growth to be readily followed. Experiments of interest were repeated with all strains and more accurate appraisal of the growth of some strains was made by colony counts. These counts were the average of counts obtained from the duplicate cultures. The final amount of growth, when required, was estimated with a Hilger Biochem Absorptiometer. Maximum yields of organisms from the medium was not sought, and no extra glutamate was added to give this.

In two experiments cultures from some strains were shaken on a Kahn shaker (Townson \& Mercer). This has a horizontal stroke of $4 \mathrm{~cm}$. and was shaken at approximately 120 strokes/min. The rheostat was removed from the body of the shaker and the tray was insulated with rubber to prevent overheating of the cultures. These experiments are more fully described below.

\section{RESULTS}

Virulence of test strains. The approximate LD 50 doses for the eight strains are given in Table 1. No mice were killed by strain $1011 \mathrm{HR}$, at the maximum dose tested $\left(20 \times 10^{6}\right.$ organisms) so this strain is called avirulent. The results 
for strains 1017 and 1018 which showed an intracerebral LD 50 of 400 and 145 organisms, respectively, indicate the considerable intracerebral virulence of these strains, while the remaining strains all showed some virulence whether given by the intracerebral or intranasal routes.

Table 1. Approximate LD 50 doses for strains of Bordetella pertussis

$\begin{array}{lcc} & \begin{array}{c}\text { Intra- } \\ \text { cerebral LD 50 } \\ \text { (in millions } \\ \text { of organisms) }\end{array} & \begin{array}{c}\text { Intra- } \\ \text { nasal LD 50 } \\ \text { (in millions } \\ \text { of organisms) }\end{array} \\ \text { Strain } & 0.2 & 0.20 \\ 1011 & 33.0 & 0 \cdot 36 \\ 1012 & 10.9 & 1 \cdot 10 \\ 1013 & 1 \cdot 1 & - \\ 1014 & 5 \cdot 4 & 0.43 \\ 1015 & 0.0004 & 6.00 \\ 1017 & 0.0015 & 0.45 \\ 1018 & >20.0 & >20.00 \\ 1011 \text { HR } & & \end{array}$

\section{Growth experiments}

Growth of test strains of pertussis organisms in basal media plus increasing quantities of magnesium sulphate. Each of the test strains was grown in basal medium containing respectively $0.048,0.096,0.24,0.48$ and $0.96 \mathrm{mg}$. $\mathrm{MgSO}_{4} 7 \mathrm{H}_{2} \mathrm{O} / \mathrm{ml}$. Visual examination of cultures showed that strains 1017 and 1018 grew more slowly with smaller quantities of magnesium sulphate than strains 1011 and 1015 and the avirulent strain $1011 \mathrm{HR}$. This was confirmed by counts of the growth of strains 1015, 1017, 1018 and $1011 \mathrm{HR}$ in media containing, respectively, 0.048 and $0.96 \mathrm{mg}$. $\mathrm{MgSO}_{4} \mathbf{z H}_{2} \mathrm{O} / \mathrm{ml}$. Strain 1015 was representative of the strains 1011 to 1015 . The results are given in Fig. 1 and show the striking improvement in growth of strains 1017 and 1018 with the larger amount of magnesium sulphate. Magnesium sulphate was therefore used in all following experiments to augment the basal medium.

Growth of test strains of pertussis organisms in basal media at $\mathrm{pH}$ values $7 \cdot 3-$ $7 \cdot 9$ and augmented by $\mathrm{MgSO}_{4} 7 \mathrm{H}_{2} \mathrm{O}$ to $0 \cdot 48 \mathrm{mg} . / \mathrm{ml}$. Rowatt (1957) found that pertussis organisms showed little variation in growth with the $\mathrm{pH}$ value of the medium. This was confirmed for most of the test strains, 1017 being the exception (see Fig. 2). The final amounts of growth obtained differed, some strains (e.g. 1015) giving a greater yield in a medium at a lower $\mathrm{pH}$ value, and some (e.g. 1018) a lesser yield.

A further experiment in which cultures of strains 1011, 1017 and 1018 were incubated on the shaker gave the results in Figs. 3 and 4. The growth of organisms obtained in 2 days from strain 1011 was considerably less from media at $\mathrm{pH} 7 \cdot 3$ than from media at $\mathrm{pH} 7 \cdot 86$, whilst not only was no growth obtained from strain 1017 in shaken culture but the death of the organisms was more rapid at $\mathbf{p H ~} \mathbf{7 \cdot 3}$ than at $\mathbf{p H} \mathbf{7 \cdot 8 6}$. Final growth determinations made by opacity measurements for all these strains are quoted in Table 2. They show the inability of strain 1017 to grow and the poor growth obtained from 


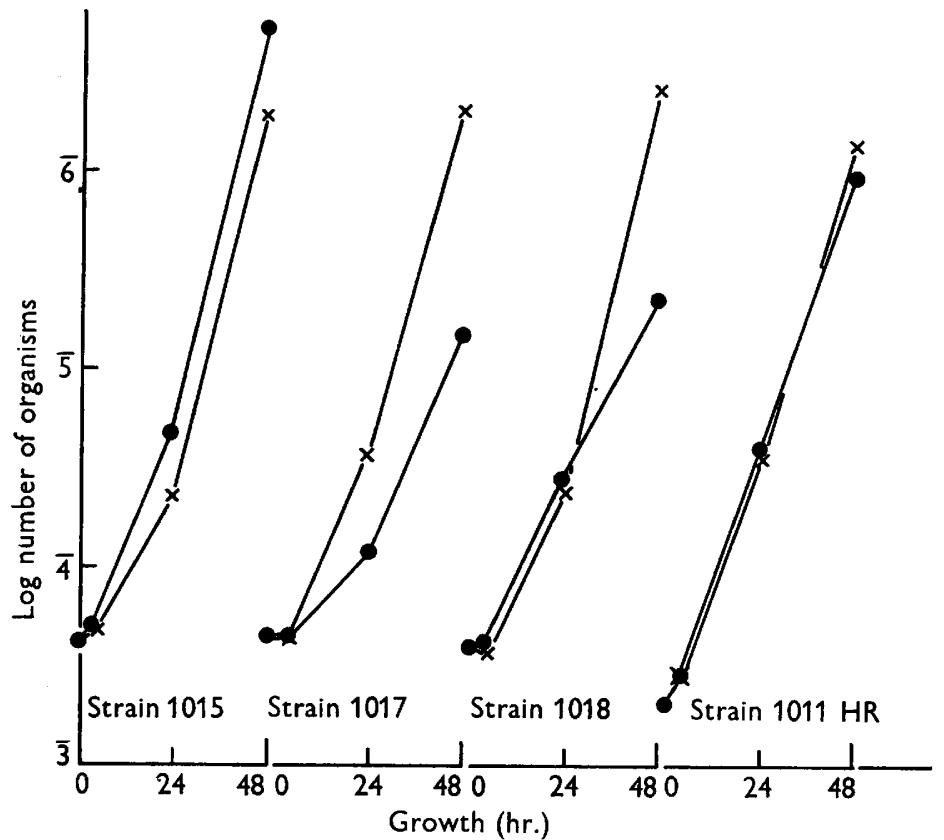

Fig. 1. Effect of concentration of magnesium sulphate on growth of four strains of Bordetella pertussis in augmented Jebb \& Tomlinson medium. $0,048 \mathrm{mg}$. $\mathrm{MgSO}_{4} 7 \mathrm{H}_{2} \mathrm{O} / \mathrm{ml}$.; $\times-\times, 0.96 \mathrm{mg} . \mathrm{MgSO}_{4} 7 \mathrm{H}_{2} \mathrm{O} / \mathrm{ml}$.

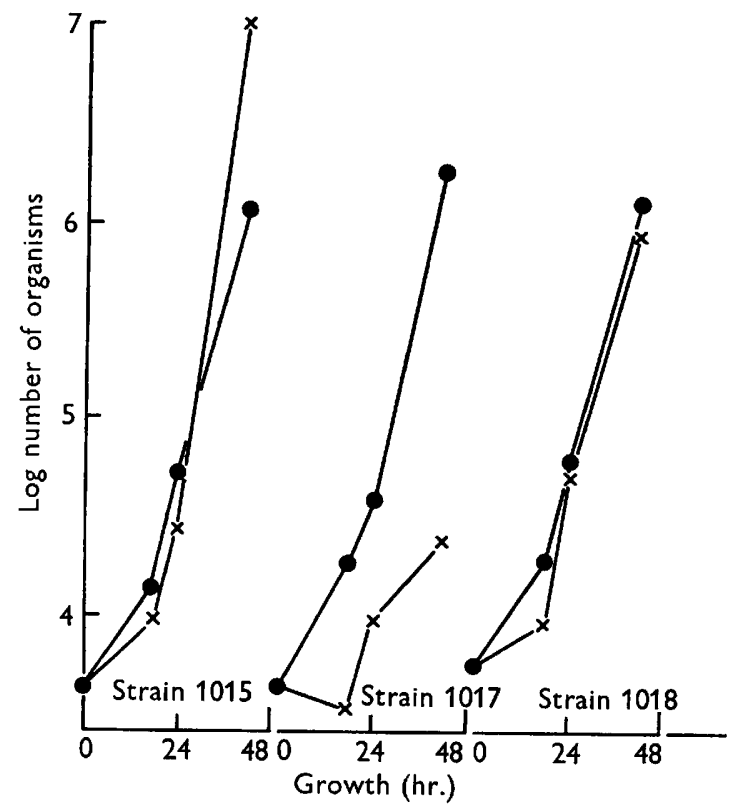

Fig. 2. Growth of Bordetella pertussis strains 1015, 1017 and 1018 at $\mathrm{pH} 7 \cdot 86$ and $7 \cdot 3$.

- , growth at $\mathrm{pH} 7 \cdot 86 ; \times-\times$, growth at $\mathrm{pH} 7 \cdot 3$. 
strain 1018 when $20 \mathrm{ml}$. cultures were shaken in $100 \mathrm{ml}$. containers, whatever the $\mathrm{pH}$ value of the medium.

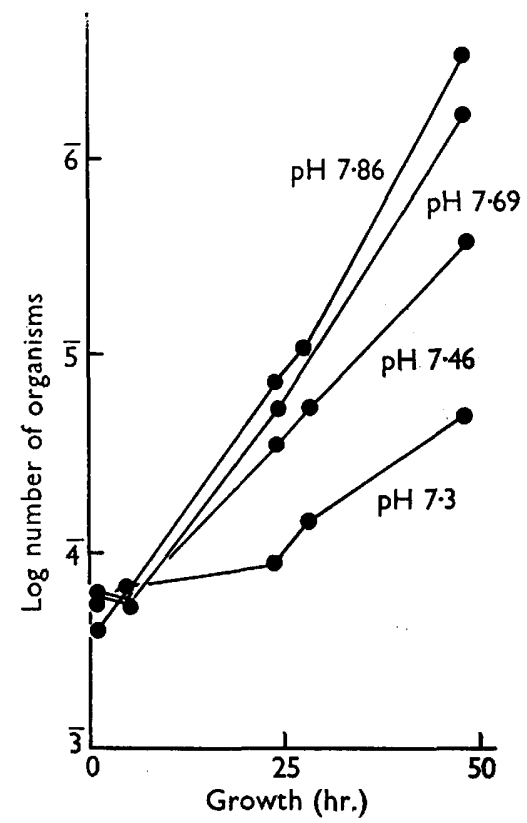

Fig. 3

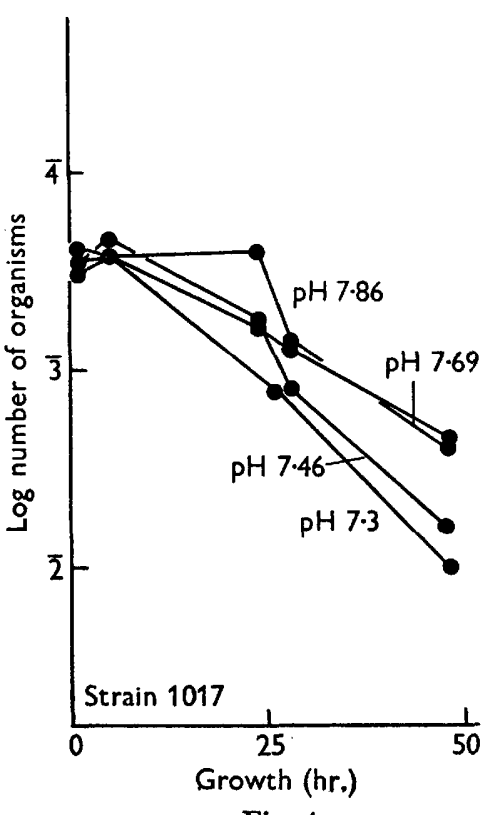

Fig. 4

Fig. 3. Growth of Bordetella pertussis strain 1011 on shaking machine in media at different pH values.

Fig. 4. Growth of Bordetella pertussis strain 1017 on shaking machine in media at different pH values.

Table 2. Measurement by \% absorption of light of final growth of Bordetella pertussis strains 1011, 1017 and 1018 in $20 \mathrm{ml}$. volumes of media at different $\mathrm{pH}$ values

The cultures were incubated at $36^{\circ}$ on a shaker in $100 \mathrm{ml}$. bottles.

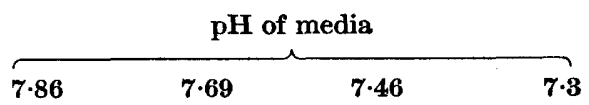

\begin{tabular}{|c|c|c|c|c|}
\hline \multirow[b]{2}{*}{ Strains } & \multicolumn{4}{|c|}{$\%$ absorption of light } \\
\hline & & & & \\
\hline 1011 & $85 \cdot 5$ & $86 \cdot 2$ & 83.5 & $81 \cdot 5$ \\
\hline 1017 & \multicolumn{4}{|c|}{ No absorption } \\
\hline 1018 & $1 \cdot 0$ & $62 \cdot 4$ & $28 \cdot 4$ & $27 \cdot 0$ \\
\hline
\end{tabular}

Growth of four of the strains of pertussis organisms in basal medium containing catalase (1 unit/ml.) to replace the usual content of haematin. Two $20 \mathrm{ml}$., two $40 \mathrm{ml}$. and two $60 \mathrm{ml}$. volumes of medium were inoculated with each of strains 1011, 1012, 1017 and 1018, and these cultures were incubated at $36^{\circ}$ on the shaker. In this experiment catalase, which was found by Mazloum \& 
Rowley (1955) to initiate the growth of pertussis organisms on solidified Cohen \& Wheeler medium (1946), was used to replace the haematin usually added. Growth appeared first in the $60 \mathrm{ml}$. volumes of medium for all strains. Slight delay only occurred in $40 \mathrm{ml}$. of medium, and strains 1011 and 1012 grew almost equally as well in $20 \mathrm{ml}$. medium. There was, however, no growth of strain 1017 in $20 \mathrm{ml}$. of medium and only slight growth in one of the $20 \mathrm{ml}$. cultures inoculated with strain 1018. This experiment was repeated with virtually the same results. It must be concluded that the conditions which obtain when $20 \mathrm{ml}$. of culture are shaken in a $100 \mathrm{ml}$. square bottle do not allow optimal growth of any of these strains, but that the growth of strains 1017 and 1018 is more sensitive to a different gas phase composition of carbon dioxide and oxygen than the other strains examined.

The growth of pertussis organisms in a control medium compared with that in a similar broth to which crystalline lysozyme has been added. The growth from eight strains of pertussis organisms in the basal medium augmented with $0 \cdot 48 \mathrm{mg}$. $\mathrm{MgSO}_{4} 7 \mathrm{H}_{2} \mathrm{O} / \mathrm{ml}$. at $c$. $\mathrm{pH} 7 \cdot 8$ was compared with that in the same medium to which crystalline lysozyme had been added. The concentration of lysozyme used in the experiment $(100 \mathrm{mg}$. $/ \mathrm{ml}$.) was approximately that present in horse sera; mouse sera contained a similar concentration, but sheep sera only $c$. one-tenth the amount. Lungs washed with saline, macerated in $10 \mathrm{ml}$. saline and the supernatant fluid titrated for lysozyme were found to contain c. $1000 \mathrm{mg}$. lysozyme/lung. Brains treated in the same way appeared to contain little. All these titrations were made with Micrococcus lysodeikticus in conjunction with crystalline lysozyme (Light \& Co.).

Counts of only four strains were made and those in the control medium are not shown as the growth of all the strains was very similar. The counts of organisms from strains 1011 and 1012 (Fig. 5) illustrate the growth of six of the test strains in the presence of lysozyme. The inhibition of the growth of strains 1017 and 1018 is shown by the decreasing number of organisms with these strains. The possibility that this difference in reaction might be due to an impurity in the lysozyme must be considered. If $1 \%$ of such an impurity were present, it would need to inhibit strains 1017 and 1018 in a concentration of $1 \mathrm{mg} . / \mathrm{ml}$. of medium. This possibility has not been investigated.

The partial reversal of the inhibition by lysozyme on the growth of pertussis organisms. The growth of four strains of pertussis organisms (1011, 1013, 1015 and 1017) in Jebb \& Tomlinson medium containing added yeast dialysate instead of nicotinamide was compared with that in a similar medium to which $100 \mathrm{mg}$. lysozyme $/ \mathrm{ml}$. had been added. Inoculation with approximately $10^{3}$ organisms of each strain was made into the control medium and into medium containing lysozyme. Weekly and twice weekly subcultures were made with a platinum loop. All strains continued to grow for 16 and 32 subcultures, respectively, in both media, although visible growth of strain 1017 was somewhat slower from the original inoculum. The inhibition noted in the previous experiment in the presence of lysozyme must have been annulled by some factor in the yeast dialysate. 


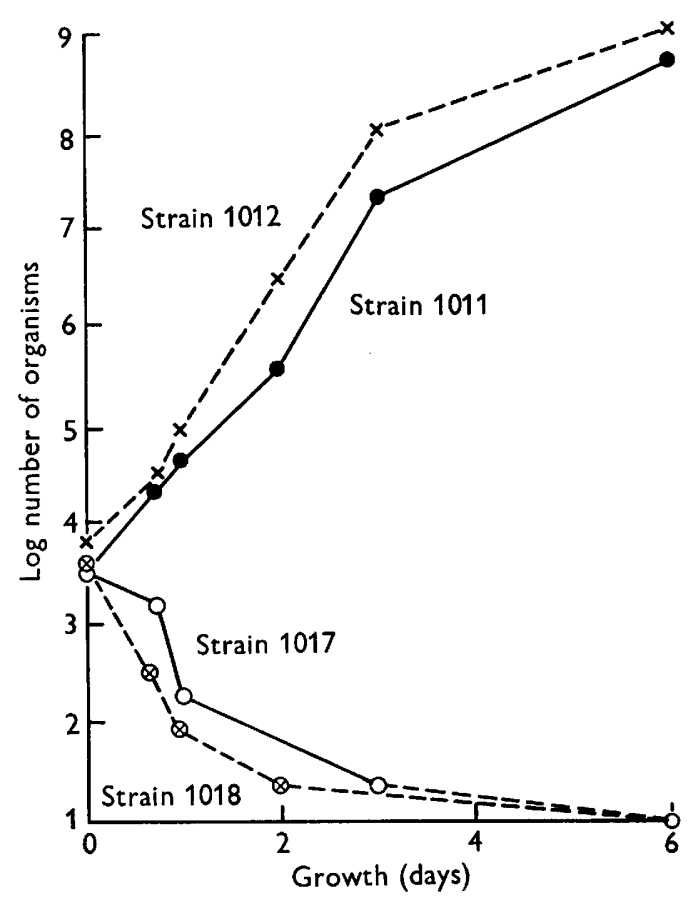

Fig. 5. Counts of organisms from growth of Bordetella pertussis strains in augmented Jebb \& Tomlinson medium $+100 \mathrm{mg}$. lysozyme/ml.

\section{DISCUSSION}

The idiosyncrasies of growth described in the previous experiments are restricted to Bordetella pertussis, strains 1017 and 1018. These strains have LD 50 of 400 and 1500 organisms, respectively, on intracerebral injection, values at least a thousand times smaller than the LD 50 doses of the other test strains. Lacey (1951) showed that $\boldsymbol{B}$. pertussis grown on solid medium containing $33 \%(\mathrm{v} / \mathrm{v})$ horse blood and magnesium sulphate instead of sodium chloride in the agar base, changed in antigenic structure. In the present work it has been shown that strains 1017 and 1018, which are very virulent when injected into mice by the intracerebral route, need more magnesium for optimal growth in the defined medium than the less virulent or avirulent strains examined. I did not examine these strains for antigenic differences from typical virulent strains. However, the avirulent degraded strain, which would be expected to differ antigenically from typical virulent strains, did not differ in its magnesium requirements from the typical virulent strains.

The inhibition of the growth of strains 1017 and 1018 by lysozyme in this particular basal medium was repeated regularly. The possibility that the inhibition is due to an impurity has not been explored, but when yeast dialysate was used as growth factor instead of nicotinamide growth was only delayed. Bordetella pertussis, strains 1011 and 1017, suspended in $1 \%$ saline containing $0 \cdot 1 \%$ sodium bicarbonate were not lysed by $1 \mathrm{mg}$. lysozyme $/ \mathrm{ml}$., as deter- 
mined by opacity measurements with the Hilger Biochem Absorptiometer. Also I failed to detect glucosamine or acetylglucosamine in the supernatant fluids from a similar suspension of strain 1011 incubated with lysozyme. It is clear that lysozyme is present in all Bordet-Gengou media (in the horse blood) in which pertussis organisms grow well. Lysozyme is also widely distributed in body tissues and fluids, although $\mathrm{I}$ failed to find it in mouse brains in which strains 1017 and 1018 grow so well from small inocula. Certain surface active substances, e.g. polymyxin, otherwise 'aerosporin' (Ainsworth, Brown \& Brownlee, 1947) and ' Tween 80' inhibit growth of typical pertussis organisms in vitro. No surface acting substance, however, has been noted which reveals a difference between those strains which are very virulent on intracerebral injection and those which are normally virulent strains. These in vitro properties are not necessarily connected with the difference in the rate of growth noted in mouse brains for strains 1011 and 1017 (Brown, 1958). It is, however, interesting that those strains more virulent when injected into mice by the intracerebral route should show the idiosyncrasies of growth described in this paper.

I would like to thank Dr L. F. Hewitt for his interest in this work and I am grateful to Mr M. Roberts for skilled technical assistance.

\section{REFERENCES}

Ainsworth, G. C., Brown, A. M. \& Brownlee, G. (1947). Aerosporin, an antibiotic produced by Bacillus aerosporin, Greer. Nature, Lond. 160, 263.

ANDERSEN, E. K. (1952). Serological studies on H. pertussis, H. para pertussis and H. bronchisepticus; preliminary report. Acta path. microbiol. scand. 30, 54.

Brown, A. M. (1958). Intracerebral infection of mice with Haemophilus pertussis and passive protection by hyperimmune rabbit sera. J. gen. Microbiol. 18, 48.

Cohen, S. M. \& Wheerer, M. W. (1946). Pertussis vaccines produced with phase I cultures grown on fluid medium. Amer. J. publ. Hlth, 36, 371.

JeBb, W. H. \& Tomlinson, A. H. (1955). The nutritional requirements of Haemophilus pertussis. J. gen. Microbiol. 13, 1.

Kendrick, P. L., Eldoering, G., Dixon, M. K. \& Misner, J. (1947). Mouse protection tests in the study of pertussis vaccine. A comparative series using the intracerebral route for challenge. Amer. J. publ. Hlth, 37, 803.

LACEY, B. W. (1951). Antigenic modulation of Haemophilus pertussis. J. gen. Microbiol. 5, xxi.

Mazloum, H. A. \& Rowley, D. (1955). The growth requirements of Haemophilus pertussis on solid media. J. Path. Bact. 70, 439.

MrLes, A. A. \& Misra, S. S. (1938). The estimation of the bactericidal power of blood. J. Hyg., Camb. 38, 732.

Rowatr, E. (1957). The growth of Bordetella pertussis: a review. J. gen. Microbiol. $17,297$. 